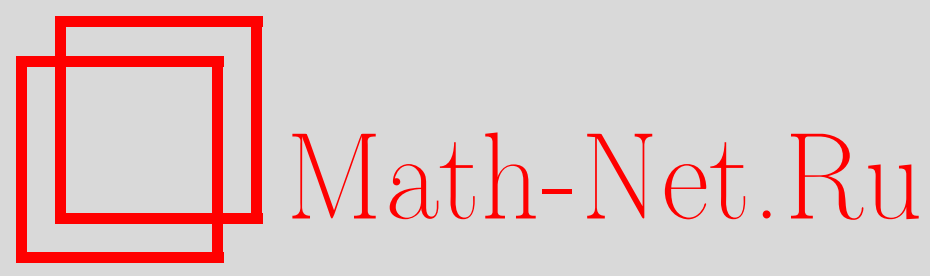

Е. В. Вахитова, Об одномерном решете Сельберга с весами Бухштаба нового типа, Матем. заметки, 1999, том 66, выпуск 1, 38-49

DOI: https://doi.org/10.4213/mzm1140

Использование Общероссийского математического портала Math-Net.Ru подразумевает, что вы прочитали и согласны с пользовательским соглашением http://www . mathnet.ru/rus/agreement

Параметры загрузки:

IP: 107.22 .136 .117

26 апреля 2023 г., 09:42:04

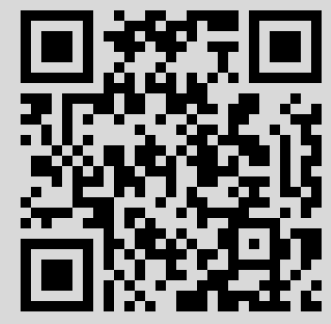




\title{
ОБ ОДНОМЕРНОМ РЕШЕТЕ СЕЛЬБЕРГА С ВЕСАМИ БУХШТАБА НОВОГО ТИПА
}

\section{Е.В. Вахитова}

\begin{abstract}
В работе изучается метод решета Сельберга с весами Бухштаба нового типа. Доказанная теорема позволяет получить преимущества в выборе параметров одномерного решета с весами по сравнению с известньми ранее результатами.

Библиографоия: 11 названий.
\end{abstract}

Введение. Метод решета, наряду с другими приложениями, успешно можно применять при решении теоретико-числовых задач, в которых простые числа заменяются числами с ограниченным количеством простых делителей. (Такие числа называются почти простыми числами). Рассмотрим конечную последовательность $A$ целых чисел $a_{n}$, где $n \in \mathbb{N}$. Через $\nu\left(a_{n}\right)$ обозначим количество простых чисел в разложении $a_{n}$ с учетом их кратности. Поставим задачу: получить оценку снизу количества элементов из $A$, имеющих в своем разложении не более $r$ простых чисел с учетом их кратности $(r \in \mathbb{N}, r \geqslant 2)$, т.е. оценку снизу количества $r$-почти простых чисел в последовательности $A$. Для решения задачи применим метод одномерного решета Сельберга с весами Бухштаба нового типа. Имеем:

$$
\sum_{\substack{a_{n} \in A \\ \nu\left(a_{n}\right) \leqslant r}} 1 \geqslant \sum_{\substack{a_{n} \in A \\ p_{n} \geqslant X^{\frac{1}{a}} \\ \nu\left(a_{n}\right) \leqslant r}} 1=\sum_{\substack{a_{n} \in A \\ p_{n} \geqslant X^{\frac{1}{a}}}} 1-\sum_{\substack{a_{n} \in A \\ p_{n} \geqslant X^{\frac{1}{a}} \\ \nu\left(a_{n}\right) \geqslant r+1}} 1,
$$

где $p_{n}$ - наименьший простой делитель числа $a_{n}$, а $X$ - достаточно большое положительное число.

Оценку сверху для последней суммы можно находить по-разному, применяя метод решета В. Бруна [1] или А. Сельберга [2]. П. Кун [3] впервые заметил, что сила метода значительно возрастет, если в методе решета Бруна элементы из последовательности $A$ снабдить определенными весами (коэффициентами). Результаты для оценок будут еще лучше при сочетании решета Сельберга и весов Куна. Проблема нахождения оптимальных весов в решете Сельберга является очень трудной проблемой. А. А. Бухштаб [4] получил процедуру выбора весов, применяя сложньй комбинаторньй набор, разбивая весь интервал на конечное число промежутков и на каждом промежутке выбирая разные веса. Полученное комбинаторное весовое решето давало лучшие результаты. Но это была громоздкая конструкция. Х.-Э. Рихерт [5] дал свою конструкцию весовой функции. М. Лаборде [6] значительно упростил веса Бухштаба: разбил интервал на 
достаточно большое число промежутков, для каждого промежутка брал свой вес и при стремлении длины промежутка к нулю получил предельную форму. Это, по-видимому, лучшее, что до сих пор сделано с весами. Веса Рихерта являются частным случаем весов Бухштаба в непрерывной форме Лаборде и получаются при выборе параметра $b=1$.

В 1985 году А. А. Бухштаб [7] анонсировал новьй тип весового решета.

Благодаря весам удалось решить ряд теоретико-числовых задач и перенести идею на конечные последовательности значений неприводимьх полиномов натуральной степени. В [8] изучено решето Сельберга с весами Рихерта, в [9] - решето Сельберга с весами Бухштаба в непрерывной форме Лаборде, в [10] - решето Бруна с весами Бухштаба нового типа. В настоящей работе изучено решето Сельберга с весами Бухштаба нового типа. Доказана основная теорема (теорема 3 ) для последовательности $A$, которая имеет преимушества в выборе параметров решета в сравнении с теоремой, полученной ранее Рихертом [5] и улучшенной автором [9].

1. Веса Бухштаба нового типа. Приведем здесь весовую функцию $T(X)$ в общем виде, о котором автору стало известно (в 1986 г.) из устного сообщения А. А. Бухштаба. А в работе [7] дана весовая функция при $g=3$

$$
\begin{aligned}
& T(X)=\frac{1}{2} \sum_{X^{\frac{1}{a}} \leqslant p<X^{\frac{a-1}{g a}}} S_{k}\left(A_{p} ; X^{\frac{1}{a}}\right)+\frac{1}{2 c-b-1} \\
& \times\left\{(c-b) \sum_{X^{\frac{a-1}{g a}} \leqslant p<X^{\frac{a-g}{a}}} S_{k}\left(A_{p} ; X^{\frac{1}{a}}\right)\right. \\
& +\sum_{X^{\frac{a-g}{a}} \leqslant p<X^{\frac{c}{a}}}\left(c-a \frac{\ln p}{\ln X}\right) S_{k}\left(A_{p} ; X^{\frac{1}{a}}\right) \\
& +a \int_{\frac{1}{a}}^{\frac{1+a(g-1)}{a g^{2}}}\left(\sum_{X^{\frac{a-1}{g a}} \leqslant p<X^{1-g z}} S_{k}\left(A_{p} ; X^{z}\right)\right) d z \\
& +\sum_{X^{\frac{1}{a}} \leqslant p<X^{\frac{a-1}{g a}}}\left(\frac{b+1}{2}-a \frac{\ln p}{\ln X}\right) S_{k}\left(A_{p} ;\left(\frac{X}{p}\right)^{\frac{1}{g}}\right) \\
& \left.+\frac{1}{g} \sum_{X^{\frac{a-1}{g a}} \leqslant p<X^{\frac{a-g}{a}}}\left(b g-a+g-a(g-1) \frac{\ln p}{\ln X}\right) S_{k}\left(A_{p} ;\left(\frac{X}{p}\right)^{\frac{1}{g}}\right)\right\},
\end{aligned}
$$

где $k \in \mathbb{N}, a, b, c \in \mathbb{R}, 1 \leqslant b \leqslant c \leqslant a, 2 c-b-1>0, S_{k}\left(A_{d} ; z\right)=\mid\left\{a_{n} \in A: a_{n} \equiv 0\right.$ $\left.(\bmod d),\left(a_{n}, P_{k}(z)\right)=1\right\} \mid, P_{k}(z)=\prod_{\substack{p<z \\ p \mid k}} p, 1 \leqslant g \leqslant a-1$.

Параметр $g$ зависит от размерности решета, в случае одномерного решета лучше выбрать $g=3$.

Лемма 1. Пусть $T(X)$ определено равенством (1), $a, b, c \in \mathbb{R}, \quad 1 \leqslant b \leqslant c \leqslant a$, 
$2 c-b-1>0,4 \leqslant a \leqslant 8, a-c \leqslant 3, g=3, k=1$. Тогда имеет место неравенство:

$$
T(X) \leqslant \sum_{\substack{a_{n} \in A \\ p_{n} \geqslant X^{\frac{1}{a}}}} \sum_{\substack{p \mid a_{n} \\ p<X^{\frac{c}{a}}}} W(p),
$$

əде

$$
W(p)=\frac{1}{2 c-b-1}\left(c-a \frac{\ln p}{\ln X}\right) .
$$

ДокАЗАТЕЛЬСтво. Преобразуем отдельно слагаемые суммы $T(X)$ из равенства (1) при $g=3, k=1$. При этом будем применять определение для $S\left(A_{d} ; z\right)$. Тогда получим, обозначив слагаемые соответственно через $Y_{i}(X), i=1,2, \ldots, 6$ :

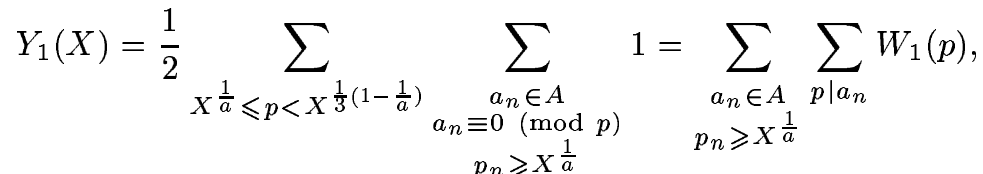

$$
\begin{aligned}
& W_{1}(p)= \begin{cases}\frac{1}{2}, & \text { если } X^{\frac{1}{a}} \leqslant p<X^{\frac{1}{3}\left(1-\frac{1}{a}\right)}, \\
0 & \text { в противном случае }\end{cases}
\end{aligned}
$$

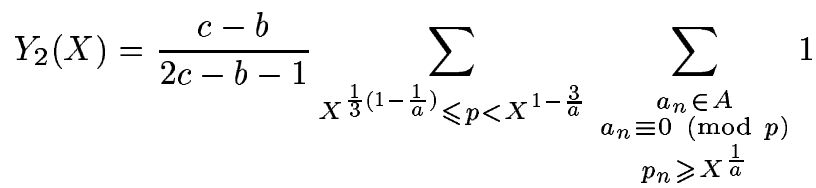

$$
\begin{aligned}
& =\sum_{\substack{a_{n} \in A \\
p_{n} \geqslant X^{\frac{1}{a}}}} \sum_{p \mid a_{n}} W_{2}(p), \\
& W_{2}(p)= \begin{cases}\frac{c-b}{2 c-b-1} x, & \text { если } X^{\frac{1}{3}\left(1-\frac{1}{a}\right)} \leqslant p<X^{1-\frac{3}{a}}, \\
0 & \text { в противном случае; }\end{cases} \\
& Y_{3}(X)=\frac{1}{2 c-b-1} \sum_{X^{1-\frac{3}{a}} \leqslant p<X^{\frac{c}{a}}}\left(c-a \frac{\ln p}{\ln X}\right) \sum_{\substack{a_{n} \in A \\
a_{n} \equiv 0(\bmod p) \\
p_{n} \geqslant X^{\frac{1}{a}}}} 1 \\
& =\sum_{a_{n} \in A} \sum_{\substack{p \mid a_{n} \\
p_{n}}} \frac{c-a \frac{\ln p}{\ln X}}{2 c-b-1}=\sum_{a_{n} \in A} \sum_{p \mid a_{n}} W_{3}(p), \\
& W_{3}(p)= \begin{cases}\frac{1}{2 c-b-1}\left(c-a \frac{\ln p}{\ln X}\right), & \text { если } X^{1-\frac{3}{a}} \leqslant p<X^{\frac{c}{a}}, \\
0 & \text { в противном случае }\end{cases}
\end{aligned}
$$

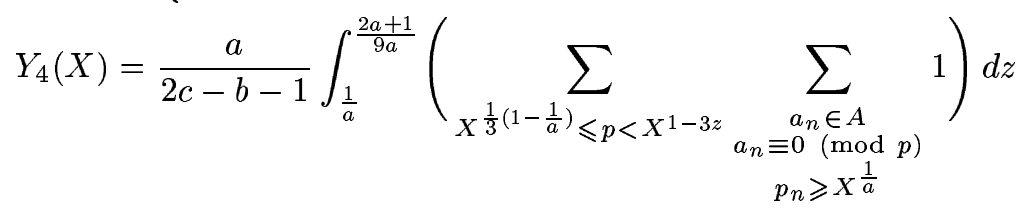




$$
=\sum_{\substack{a_{n} \in A \\ p_{n} \geqslant X^{\frac{1}{a}}}} \int_{\frac{1}{a}}^{\frac{2 a+1}{9 a}} \frac{a}{2 c-b-1}\left(\sum_{X^{\frac{1}{3}\left(1-\frac{1}{a}\right)} \leqslant p<X^{1-3 z}} 1\right) d z,
$$

так как $X^{z} \geqslant X^{\frac{1}{a}}$. Преобразуем теперь интеграл, обозначив его через $J$ :

$$
J=\int_{\frac{1}{a}}^{\frac{2 a+1}{9 a}}\left(\sum_{\substack{p \mid a_{n} \\ p_{n} \geqslant X^{z} \\ p<X^{1-3 z}}} \frac{a}{2 c-b-1}\right) d z-\int_{\frac{1}{a}}^{\frac{2 a+1}{9 a}}\left(\sum_{\substack{p \mid a_{n} \\ p_{n} \geqslant X^{z} \\ p<X^{1}\left(1-\frac{1}{a}\right)}} \frac{a}{2 c-b-1}\right) d z .
$$

Для первого интеграла получим, что

$$
z \leqslant \min \left(\frac{\ln p_{n}}{\ln X} ; \frac{1}{3}\left(1-\frac{\ln p}{\ln X}\right)\right),
$$

а отсюда следует, что $z \leqslant(2 a+2) /(9 a)$ при $a \leqslant 8$. Поэтому для $Y_{4}(X)$ получим неравенство:

$$
\begin{aligned}
& Y_{4}(X) \leqslant \sum_{\substack{a_{n} \in A \\
p_{n} \geqslant X^{\frac{1}{a}}}}\left(\sum_{\substack{p<X^{1-\frac{3}{a}} \\
p \mid a_{n}}} \int_{\frac{1}{a}}^{\min \left(\frac{\ln p_{n}}{\ln X} ; \frac{1}{3}\left(1-\frac{\ln p}{\ln X}\right)\right)} \frac{a}{2 c-b-1} d z\right. \\
& \left.-\sum_{\substack{p<X^{\frac{1}{3}\left(1-\frac{1}{a}\right)} \\
p \mid a_{n}}} \int_{\frac{1}{a}}^{\frac{\ln p_{n}}{\ln X}} \frac{a}{2 c-b-1} d z\right)=\sum_{\substack{a_{n} \in A \\
p_{n} \geqslant X^{\frac{1}{a}}}} \sum_{p \mid a_{n}} W_{4}(p),
\end{aligned}
$$

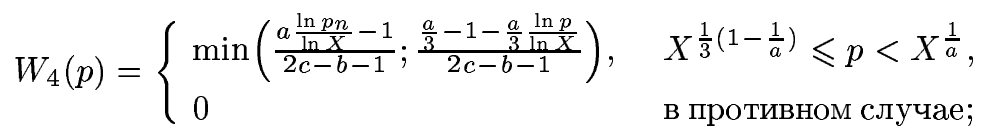

$$
\begin{aligned}
& Y_{5}(X)=\sum_{X^{\frac{1}{a} \leqslant p<X^{\frac{1}{3}}\left(1-\frac{1}{a}\right)}} \frac{1}{2 c-b-1}\left(\frac{b+1}{2}-a \frac{\ln p}{\ln X}\right) \sum_{\substack{a_{n} \in A \\
a_{n} \equiv(\bmod p)}} 1 . \\
& p_{n} \geqslant\left(\frac{X}{p}\right)^{\frac{1}{3}}
\end{aligned}
$$

Если $p \mid a_{n}, X^{\frac{1}{a}} \leqslant p<X^{\frac{1}{3}\left(1-\frac{1}{a}\right)}$, и $p_{n} \geqslant\left(\frac{X}{p}\right)^{\frac{1}{3}}$, то отсюда получим, что $p_{n} \geqslant X^{\frac{2 a+1}{9 a}}$, а следовательно, $p_{n} \geqslant X^{\frac{1}{a}}$. Тогда

$$
\begin{aligned}
& Y_{5}(X)=\sum_{\substack{a_{n} \in A \\
p_{n} \geqslant X^{\frac{1}{a}}}} \sum_{p \mid a_{n}} W_{5}(p), \\
& W_{5}(p)= \begin{cases}\frac{1}{2 c-b-1}\left(\frac{b+1}{2}-a \frac{\ln p}{\ln X}\right), & X^{\frac{1}{a}} \leqslant p<X^{\frac{1}{3}\left(1-\frac{1}{a}\right)}, \\
0 & \text { в противном случае; }\end{cases} \\
& Y_{6}(X)=\frac{1}{3(2 c-b-1)} \sum_{X^{\frac{1}{3}\left(1-\frac{1}{a}\right)} \leqslant p<X^{1-\frac{3}{a}}}\left(3 b-a+3-2 a \frac{\ln p}{\ln X}\right)
\end{aligned}
$$




$$
\begin{gathered}
\times \sum_{\substack{a_{n} \in A \\
a_{n} \equiv 0(\bmod p) \\
p_{n} \geqslant\left(\frac{X}{p}\right)^{\frac{1}{3}}}} 1=\sum_{\substack{a_{n} \in A \\
p_{n} \geqslant X^{\frac{1}{a}}}} \sum_{p \mid a_{n}} W_{6}(p), \\
W_{6}(p)= \begin{cases}\frac{1}{2 c-b-1}\left(b+1-\frac{a}{3}\left(1+2 \frac{\ln p}{\ln X}\right)\right), & X^{\frac{1}{3}\left(1-\frac{1}{a}\right)} \leqslant p<X^{1-\frac{3}{a}}, \\
0 & \text { в противном случае. }\end{cases}
\end{gathered}
$$

Таким образом, если обозначим теперь через $W(p)$ сумму $\sum_{i=1}^{6} W_{i}(p)$, то послепреобразований для $W(p)$ получим равенство $(3)$, а для $T(X)$ - неравенство (2). Лемма 1 доказана.

Введем следующее условие.

Существует некоторая постоянная $M$ такая, что

$$
\left|a_{n}\right| \leqslant X^{M}
$$

для всех $a_{n}$ из последовательности $A$.

Теорема 1. Пусть $T(X)$ определено равенством $(1)$, выполнено условие $\left(u_{0}\right) u$ $(r+1) c-M a=2 c-b-1,2 c-b-1>0,1 \leqslant b \leqslant c \leqslant a, 4 \leqslant a \leqslant 8, a-c \leqslant 3, g=3$, $k=1$. Тогда имеет место неравенство:

$$
\sum_{\substack{a_{n} \in A \\ \nu\left(a_{n}\right) \leqslant r}} 1 \geqslant S\left(A ; X^{\frac{1}{a}}\right)-T(X)-R,
$$

где $R$ - число әлементов из $A$, делящихся на квадрат простого числа $р$ из интервала $X^{\frac{1}{a}} \leqslant p<X^{\frac{c}{a}}$.

ДокАЗАТЕльство. Применим лемму 1. Тогда, учитывая определение $S\left(A ; X^{\frac{1}{a}}\right)$, получим, что

$$
S\left(A ; X^{\frac{1}{a}}\right)-T(X) \geqslant \sum_{\substack{a_{n} \in A \\ p_{n} \geqslant X^{\frac{1}{a}}}} 1-\sum_{\substack{a_{n} \in A \\ p_{n} \geqslant X^{\frac{1}{a}}}} \sum_{\substack{p \mid a_{n} \\ p<X^{\frac{c}{a}}}} W(p)
$$

где $W(p)$ определено равенством (3).

Но последняя разность будет положительной. Кроме того,

$$
\sum_{\substack{a_{n} \in A \\ \nu\left(a_{n}\right) \leqslant r}} 1 \geqslant \sum_{\substack{a_{n} \in A \\ p_{n} \geqslant X^{\frac{1}{a}}}} 1-\sum_{\substack{a_{n} \in A \\ p_{n} \geqslant X^{\frac{1}{a}} \\ \nu\left(a_{n}\right) \leqslant r+1}} 1
$$

Теорема 1 доказана. 
2. Одномерное решето Сельберга с весами Бухштаба нового типа. Приведем полученньй результат в случае одномерного решета к форме, удобной для приложений. Для этого введем несколько условий.

1) Существует постоянная $A_{1} \geqslant 1$ такая, что

$$
1 \leqslant\left(1-\frac{\omega(p)}{p}\right)^{-1} \leqslant A_{1}
$$

для любого простого числа $p$, где $\omega(p)$ - такое, что $\omega(d) / d X$ является приближением $\left|A_{d}\right|$ и $\mu(d) \neq 0\left(\mu(n)\right.$ - функция Мебиуса). Вьполнимость условия $\left(u_{1}\right)$ дает возможность рассматривать

$$
\prod_{p}\left(1-\frac{\omega(p)}{p}\right)
$$

2) Существуют постоянная $A_{2} \geqslant 1$ и параметр $L$ такие, что

$$
-L \leqslant \sum_{v \leqslant p<\omega} \frac{\omega(p)}{p} \ln p-\ln \frac{\omega}{v} \leqslant A_{2},
$$

где $L \geqslant 1$ и не зависит от $v$ и $\omega(2 \leqslant v \leqslant \omega)$.

Условие $\left(u_{2}\right)$ говорит о том, что $\omega(p)$, по крайней мере в среднем по $p$, равно 1 , иначе будем рассматривать только те последовательности $A$, при просеивании которых возникает задача одномерного решета.

3) Существуют постоянные $\alpha(0<\alpha \leqslant 1), A_{3} \geqslant 1, A_{5} \geqslant 1$ такие, что

$$
\sum_{d<\frac{X^{\alpha}}{\ln ^{A_{5} X}}} \mu^{2}(d) 3^{\nu(d)}|R(X, d)| \leqslant A_{3} \frac{X}{\ln ^{C} X}
$$

где $X \geqslant 2, A_{3}=A_{3}(C), R(X, d)=\left|A_{d}\right|-\omega(d) X / d$. Условие $\left(u_{3}\right)$ позволяет говорить о достаточной малости остаточного члена $R(X, d)$.

4) Существует постоянная $A_{4} \geqslant 1$ такая, что

$$
\sum_{z \leqslant p<y} \sum_{\substack{a_{n} \in A \\ a_{n} \equiv 0\left(\bmod p^{2}\right)}} 1 \leqslant A_{4}\left(\frac{X \ln X}{z}+y\right)
$$

если $2 \leqslant z \leqslant y \leqslant X$.

Условие $\left(u_{4}\right)$ необходимо для того, чтобы можно было нужным образом оценить величину $R$, входящую в неравенство теоремы 1 .

Кроме того, пусть вьполнены условия: $c<\alpha a, 2<\alpha a$, где $\alpha$ берется из условия $\left(u_{3}\right)$.

Предположим, что 1$)$ при некоторой постоянной $\delta(0<\delta \leqslant 1)$ для $S\left(A ; X^{\frac{1}{a}}\right)$ имеет место следующая оценка снизу [8, теорема 7.4]:

$$
S\left(A ; X^{\frac{1}{a}}\right) \geqslant X \cdot W\left(X^{\frac{1}{a}}\right)\left(f(\alpha a)-C_{1} \frac{L}{\ln ^{\delta} X}\right),
$$


где $f(\alpha a)>0$,

$$
W(z)=\prod_{p<z}\left(1-\frac{\omega(p)}{p}\right)
$$

2) для любого действительного числа $\xi, \xi>1$, удовлетворяющего неравенствам

$$
\frac{1}{C_{2}} \leqslant a \frac{\ln \xi^{2}}{\ln X} \leqslant \alpha a-1,
$$

для всех простых $p$ из интервала $\left[X^{\frac{1}{a}} ; X^{\frac{c}{a}}\right)$ для $S\left(A_{p} ; X^{\frac{1}{a}}\right)$ имеет место следующая оценка сверху [8, теорема 8.3]:

$$
\begin{aligned}
S\left(A_{p} ; X^{\frac{1}{a}}\right) \leqslant & \frac{\omega(p)}{p} X W\left(X^{\frac{1}{a}}\right)\left(F\left(a \frac{\ln \xi^{2}}{\ln X}\right)+C_{3} \frac{L}{\ln ^{\delta} \xi}\right) \\
& +C_{4} \sum_{\substack{d<\xi^{2} \\
d \mid P\left(X^{\frac{1}{a}}\right)}} 3^{\nu(d)}|R(X, \rho d)|
\end{aligned}
$$

где для $u>0$ функция $F(u)$ является неотрицательной, убьвающей и непрерьвной, удовлетворяющей условию:

$$
F\left(u_{1}\right)-F\left(u_{2}\right) \leqslant C_{5} \frac{u_{2}-u_{1}}{u_{1}}, \quad \frac{1}{C_{2}} \leqslant u_{1}<u_{2} .
$$

Будем считать, что $X>C_{6}$, где $C_{6}$ - достаточно большое число. Выберем $\xi^{2}$ из учета условия $\left(u_{3}\right): \xi^{2}=X^{\alpha} /\left(\ln ^{A_{5}} X\right)$. Неравенство (6) будем применять с $\xi^{2} / p$ вместо $\xi^{2}$ (это можно доказать). Проведем еще замену $\xi^{2}$ на $X^{\alpha}$ в аргументе функции $F$. Тогда

$$
\Delta F=F\left(\frac{\ln \frac{\xi^{2}}{p}}{\ln X^{\frac{1}{a}}}\right)-F\left(\frac{\ln \frac{X^{\alpha}}{p}}{\ln X^{\frac{1}{a}}}\right) \leqslant C_{7} \frac{\ln \ln X}{\ln X} .
$$

ЛЕмма 2. Пусть выполнены условия $\left(u_{1}\right)-\left(u_{4}\right),(6)$ и $T(X)$ определено равенством (1) при $g=3, k=1$. Тогда для $T(X)$ имеет место следующее неравенство:

$$
T(X) \leqslant X \cdot W\left(X^{\frac{1}{a}}\right)\left(B(\alpha, a, b, c)+\frac{C_{28} \ln \ln 3 X}{\ln ^{\delta} X}\right),
$$

где $B(\alpha, a, b, c)$ определено равенством:

$$
\begin{aligned}
B(\alpha, a, b, c)=\frac{1}{2} & \int_{\frac{2 \alpha a+1}{3}}^{\alpha a-1} \frac{F(z) d z}{\alpha a-z}+\frac{1}{2 c-b-1}\left\{(c-b) \int_{3}^{\frac{2 \alpha a+1}{3}} \frac{F(z) d z}{\alpha a-z}\right. \\
& +\int_{\alpha a-c}^{3} F(z) \frac{z-(\alpha a-c)}{\alpha a-z} d z+\int_{\frac{9 \alpha a}{2 \alpha a+1}}^{\alpha a}\left(\int_{3}^{\frac{2 \alpha a+1}{3 \alpha a} v} F(z) \frac{d z}{v-z}\right) \frac{d v}{v} \\
& +\frac{3}{2 \alpha a} \int_{\frac{2 \alpha a+1}{\alpha a-1}}^{\alpha a-1} F(3) \frac{(b+1) z-(2 \alpha a-b-1)}{z(1+z)} d z \\
& \left.+\frac{3}{\alpha a} \int_{\frac{3}{\alpha a-3}}^{\frac{2 \alpha a+1}{\alpha a-1}} F(3) \frac{\left(b+1-\frac{\alpha a}{3}\right) z-(\alpha a-b-1)}{z(1+z)} d z\right\}
\end{aligned}
$$


ДокАЗАТЕЛЬСтво. Преобразуем отдельно слагаемые суммы $T(X)$, применяя неравенство (6):

$$
\begin{aligned}
J_{1}(X) & =\frac{1}{2} \sum_{X^{\frac{1}{a}} \leqslant p<X^{\frac{1}{3}\left(1-\frac{1}{a}\right)}} S\left(A_{p} ; X^{\frac{1}{a}}\right) \\
& \leqslant X W\left(X^{\frac{1}{a}}\right)\left(\int_{\frac{2 \alpha a+1}{3}}^{\alpha a-1} \frac{1}{2} \frac{F(z) d z}{\alpha a-z}+\frac{C_{19}^{\prime} \ln \ln 3 X}{\ln ^{\delta} X}\right) ; \\
J_{2}(X) & =(c-b) \sum_{X^{\frac{1}{3}\left(1-\frac{1}{a}\right)} \leqslant p<X^{1-\frac{3}{a}}} S\left(A_{p} ; X^{\frac{1}{a}}\right) \\
& \leqslant X W\left(X^{\frac{1}{a}}\right)\left((c-b) \int_{3}^{\frac{2 \alpha a+1}{3}} \frac{F(z) d z}{\alpha a-z}+\frac{C_{19} \ln \ln 3 X}{\ln ^{\delta} X}\right) .
\end{aligned}
$$

Для третьего слагаемого $J_{3}(X)$ из $T(X)$, учитьвая, что $\Delta F \leqslant C_{7} \ln \ln X / \ln X$, получим:

$$
\begin{aligned}
J_{3}(X)= & \sum_{X^{1-\frac{3}{a}} \leqslant p<X^{\frac{c}{a}}}\left(c-a \frac{\ln p}{\ln X}\right) S\left(A_{p} ; X^{\frac{1}{a}}\right) \\
\leqslant & X W\left(X^{\frac{1}{a}}\right) \sum_{X^{\frac{a-3}{a} \leqslant p<X^{\frac{c}{a}}}}\left(\left(c-a \frac{\ln p}{\ln X}\right) \frac{\omega(p)}{p} F\left(a \frac{\ln \frac{X^{\alpha}}{p}}{\ln X}\right)\right. \\
& \left.+C_{8} \frac{\omega(p)}{p} \frac{\ln \ln 3 X}{\ln ^{\delta} X}\right)+C_{4} \sum_{X^{\frac{a-3}{a}} \leqslant p<X^{\frac{c}{a}}} \sum_{\substack{d<\frac{\xi^{2}}{p} \\
d \mid P\left(X^{\frac{1}{a}}\right)}} 3^{\nu(d)}|R(X, p d)| .
\end{aligned}
$$

Применим теперь условие $\left(u_{3}\right)$ и оценку $(2.3 .6)$ из [8] (при $k=1$ ), а затем - неравенство (5.2.1) из [8] (при $k=1$ ) и условие $\left(u_{2}\right)$. Тогда, наконец, применяя лемму Абеля о частном суммировании, получим, что

$$
\begin{aligned}
J_{3}(X) \leqslant & X W\left(X^{\frac{1}{a}}\right)\left(\int_{X^{\frac{a-3}{a}}}^{X^{\frac{c}{a}}} E(\omega)\left(c-a \frac{\ln \omega}{\ln X}\right) \frac{d \omega}{\omega \ln \omega}\right. \\
& \left.+O\left(\frac{C_{13} \ln \ln 3 X}{\ln X}\right)+\frac{C_{10} \ln \ln 3 X}{\ln ^{\delta} X}\right),
\end{aligned}
$$

где $E(\omega)=F\left(a \ln \left(X^{\alpha} / \omega\right) / \ln X\right)$. Сделаем замену переменной:

$$
z=\frac{\ln \frac{X^{\alpha}}{\omega}}{\ln X^{\frac{1}{a}}}
$$

Тогда

$$
\int_{X \frac{a-3}{a}}^{X^{\frac{c}{a}}} E(\omega)\left(c-a \frac{\ln \omega}{\ln X}\right) \frac{d \omega}{\omega \ln \omega} \leqslant \int_{\alpha a-c}^{3} F(z) \frac{z-(\alpha a-c)}{\alpha a-z} d z .
$$


Таким образом,

$$
J_{3}(X) \leqslant X W\left(X^{\frac{1}{a}}\right)\left(\int_{\alpha a-c}^{3} F(z) \frac{z-(\alpha a-c)}{\alpha a-z} d z+\frac{C_{14} \ln \ln 3 X}{\ln ^{\delta} X}\right) .
$$

Для четвертого слагаемого $J_{4}(X)$ из $T(X)$ получим

$$
\begin{aligned}
J_{4}(X)= & a \int_{\frac{1}{a}}^{\frac{2 a+1}{9 a}}\left(\sum_{X^{\frac{1}{3}\left(1-\frac{1}{a}\right)} \leqslant p<X^{1-3 z}} S\left(A_{p} ; X^{z}\right)\right) d z \\
\leqslant & a \int_{\frac{1}{a}}^{\frac{2 a+1}{9 a}}\left(\sum _ { X ^ { \frac { a - 1 } { 3 a } \leqslant p < X ^ { 1 - 3 z } } } \left(\frac{\omega(p)}{p} X W\left(X^{z}\right)\left(F\left(\frac{\ln \frac{\xi^{2}}{p}}{\ln X^{z}}\right)+\frac{C_{3} \ln \ln 3 X}{\ln ^{\delta} \frac{\xi}{\sqrt{p}}}\right)\right.\right. \\
& \left.\left.+C_{4} \sum_{\substack{d<\frac{\xi^{2}}{p} \\
d \mid P\left(X^{z}\right)}} 3^{\nu(d)}|R(X, p d)|\right)\right) d z \\
\leqslant & a \int_{\frac{1}{a}}^{\frac{2 a+1}{9 a}} X W\left(X^{z}\right)\left(\sum_{X^{\frac{a-1}{3 a}} \leqslant p<X^{1-3 z}} \frac{\omega(p)}{p} F\left(\frac{\ln \frac{X^{\alpha}}{p}}{\ln X^{z}}\right)+C_{20} \frac{\ln \ln 3 X}{\ln ^{\delta} X}\right) d z
\end{aligned}
$$

Вьполняя дальнейшие преобразования и учитывая неравенство (2.3.5) из [8], получим окончательно

$$
\begin{aligned}
J_{4}(X) \leqslant & X W\left(X^{\frac{1}{a}}\right)\left(\int_{\frac{9 \alpha a}{2 \alpha a+1}}^{\alpha a}\left(\int_{3}^{\frac{2 \alpha a+1}{3 \alpha a} v} F(z) \frac{d z}{v-z}\right) \frac{d v}{v}+\frac{C_{22} \ln \ln 3 X}{\ln ^{\delta} X}\right) \\
J_{5}(X)= & \sum_{X^{\frac{1}{a}} \leqslant p<X^{\frac{1}{3}\left(1-\frac{1}{a}\right)}}\left(\frac{b+1}{2}-a \frac{\ln p}{\ln X}\right) S_{p}\left(A_{p} ;\left(\frac{X}{p}\right)^{\frac{1}{3}}\right) \leqslant X W\left(X^{\frac{1}{a}}\right) \\
& \times\left(\frac{3}{2 \alpha a} \int_{\frac{2 \alpha a+1}{\alpha a-1}}^{\alpha a-1} F(3) \frac{(b+1) z-(2 \alpha a-b-1)}{z(1+z)} d z+\frac{C_{27} \ln \ln 3 X}{\ln ^{\delta} X}\right) \\
J_{6}(X)= & \frac{1}{3} \sum_{X^{\frac{1}{3}\left(1-\frac{1}{a}\right)} \leqslant p<X^{1-\frac{3}{a}}}\left(3 b-a+3-2 a \frac{\ln p}{\ln X}\right) S\left(A_{p} ;\left(\frac{X}{p}\right)^{\frac{1}{3}}\right) \leqslant X W\left(X^{\frac{1}{a}}\right) \\
& \times\left(\frac{3}{\alpha a} \int_{\frac{3}{\alpha a-3}}^{\frac{2 \alpha a+1}{\alpha a-1}} F(3) \frac{\left(b+1-\frac{\alpha a}{3}\right) z-(\alpha a-b-1)}{z(1+z)} d z+\frac{C_{27}^{\prime} \ln \ln 3 X}{\ln ^{\delta} X}\right)
\end{aligned}
$$

Таким образом, определяя величину $B(\alpha, a, b, c)$ равенством (7), из неравенств (8) - (13) получим требуемое неравенство для $T(X)$. Лемма 2 доказана. 
ТЕОРема 2. Пусть выполнены условия $\left(u_{1}\right)-\left(u_{4}\right),\left(u_{6}\right),(5),(6)$ и пусть $a, b, c \in \mathbb{R}$, $1 \leqslant b<c<\alpha a, 4 \leqslant \alpha a \leqslant 8, \quad \alpha a-c \leqslant 3, \quad(r+1) c-M a=2 c-b-1,2 c-b-1>0$. Тогда имеет место оченка снизу:

$$
\sum_{\substack{a_{n} \in A \\ \nu\left(a_{n}\right) \leqslant r}} 1 \geqslant X W\left(X^{\frac{1}{a}}\right)\left(f(\alpha a)-B(\alpha, a, b, c)-\frac{C_{31} \ln \ln 3 X}{\ln ^{\delta} X}\right),
$$

где $B(\alpha, a, b, c)$ определено равенством (7).

ДокаЗАТЕЛЬСтво. Применим последовательно теорему 1 , неравенство (5) и лемму 2. Тогда получим:

$$
\begin{aligned}
\sum_{\substack{a_{n} \in A \\
\nu\left(a_{n}\right) \leqslant r}} 1 & \geqslant S\left(A ; X^{\frac{1}{a}}\right)-T(X)-R \\
& \geqslant X W\left(X^{\frac{1}{a}}\right)\left(f(\alpha a)-B(\alpha, a, b, c)-\frac{C_{29} \ln \ln 3 X}{\ln ^{\delta} X}\right)-R,
\end{aligned}
$$

где $R$ - число элементов последовательности $A$, делящихся на квадрат простого числа из интервала $\left[X^{\frac{1}{a}} ; X^{\frac{c}{a}}\right)$. Оценивая теперь $R$ с учетом условия $\left(u_{4}\right)$, получим утверждение теоремы. Теорема 2 доказана.

Уточним теорему 2 , учитывая выбор функций $f(z)$ и $F(z)$. Рассмотрим функции $\eta(u)$ и $\sigma(u)$, которые определяются следуюшим образом:

$$
\sigma(u)=\frac{u \bar{e}^{\gamma}}{2}, \quad 0 \leqslant u \leqslant 2,
$$

где $\gamma$ - постоянная Эйлера,

$$
u \sigma^{\prime}(u)=\sigma(u)-\sigma(u-2), \quad u>2,
$$

причем $\sigma(u)$ непрерьвна при $u=2$,

$$
\eta(u)=\frac{1}{u} \int_{u}^{\infty}\left(\sigma^{-1}(t-1)-1\right) d t, \quad u>1 .
$$

О свойствах этих функций можно узнать из [8].

Условие $c<\alpha a, 2<\alpha a$ влечет условие (7.6.1) теоремы 7.4 из [8] (при $a=v, c=v / u$ ), а теорема 7.4 дает неравенство (5) с $f(\alpha a)=1-\eta(\alpha a)$.

Условие $f(\alpha a)>0$ означает условие $\alpha a>2$, поэтому получим условие на $a: a>2 / \alpha$.

Неравенство (6) можно получить по теореме 6.3 из [8] с $F(t)=\sigma^{-1}(t)$, причем при таком выборе $F(t)$ будет выполнено условие на $F(t)$ (учитывая (6.3.3), (6.3.4) и (6.3.5) из [8]).

В силу теорем 7.4 и 6.3 из [8] величина $\delta$ может быть любой положительной постоянной, меньшей 1 (а сами эти теоремы доказаны методом решета Сельберга).

Наконец, в дальнейшем вместо условия $\left(u_{2}\right)$ будем использовать другое условие:

$$
-A_{2} \ln \ln 3 X \leqslant \sum_{v \leqslant p \leqslant w} \frac{\omega(p)}{p} \ln p-\ln \frac{\omega}{v} \leqslant A_{2} .
$$

Уточним теперь теорему 2 , учитьвая условие $\left(u_{2}^{\prime}\right)$ и выбор функций $f(z)$ и $F(z)$. 
ТеОрема 3. Пусть выполнены условия $\left(u_{1}\right),\left(u_{2}^{\prime}\right),\left(u_{3}\right),\left(u_{4}\right),\left(u_{0}\right), f(\alpha a)=1-$ $\eta(\alpha a), \quad F(z)=\sigma^{-1}(z)$ u nycmb $a, b, c \in \mathbb{R}, \quad 1 \leqslant b<c<\alpha a, 4 \leqslant \alpha a \leqslant 8, \alpha a-c \leqslant 3$, $(r+1) c-M a=2 c-b-1,2 c-b-1>0$. Тогда имеет место следуюшая оченка снизу:

$$
\sum_{\substack{a_{n} \in A \\ \nu\left(a_{n}\right) \leqslant r}} 1 \geqslant X W\left(X^{\frac{1}{a}}\right)\left(f(\alpha a)-B(\alpha, a, b, c)-\frac{C_{28} L}{\ln ^{1 / 2} X}\right)
$$

где $B(\alpha, a, b, c)$ определено равенством (7).

Доказательство теоремы получим из теоремы 2 , учитывая условие $\left(u_{2}^{\prime}\right)$ и выбирая $\delta=1 / 2+\varepsilon, \varepsilon>0$.

Таким образом, теорема 3 позволяет получить преимущества в выборе параметров одномерного решета с весами по сравнению с известными ранее результатами.

3. Приложение решета Сельберга с весами Бухштаба нового типа. Рассмотрим последовательность $A_{1}$ :

$$
A_{1}=\{\Phi(p q): p, q-\text { простые, } p \neq q,(p q, \Phi(0))=1, p q \leqslant x\}
$$

где $\Phi(n)$ - неприводимый полином натуральной степени $g$ с целыми коэффициентами, а $x$ - достаточно большое положительное число.

ТЕорема 4. Пусть последовательность $A_{1}$ определена условием (14), выполнены условия $\left(u_{1}\right),\left(u_{2}^{\prime}\right),\left(u_{3}\right),\left(u_{4}\right),\left(u_{0}\right), f(\alpha a)=1-\eta(\alpha a), F(z)=\sigma^{-1}(z)$ и пусть $a, b, c \in \mathbb{R}, \quad 1 \leqslant b<c<\alpha a, \quad 4 \leqslant \alpha a \leqslant 8, \quad \alpha a-c \leqslant 3, \quad(r+1) c-M a=2 c-b-1$, $2 c-b-1>0$. Тогда имеет место следующая оченка снизу:

$$
\begin{aligned}
& \sum_{\substack{\Phi(p q) \in A_{1} \\
\nu(\Phi(p q)) \leqslant r}} 1 \geqslant \frac{a \bar{e}^{\gamma}}{2}(f(\alpha a)-B(\alpha, a, b, c)) \\
& \quad \times \prod_{p \nmid \Phi(0)} \frac{1-\frac{\rho(p)}{p-1}}{1-\frac{1}{p}} \prod_{p \mid \Phi(0)} \frac{1-\frac{\rho(p)-1}{p-1}}{1-\frac{1}{p}} \frac{x}{\ln ^{2} x} \ln \ln x
\end{aligned}
$$

для достаточно больиих $x$, где $B(\alpha, a, b, c)$ определено равенством $(7)$, a $\rho(d)-$ число решений сравнения $\Phi(n) \equiv 0(\bmod d)$.

ДокАЗАТЕЛЬСТво теоремы 4 получим, применяя теорему 3 и лемму 4 работы [11].

Теорема 4 позволяет получить преимушества в выборе параметров одномерного решета с весами по сравнению с ранее доказанной соответствующей теоремой в работе [9] (с помощью метода решета Сельберга с весами Бухштаба в непрерывной форме Лаборде).

Автор благодарит профессора Н.М. Тимофеева за полезные обсуждения. 


\section{СПИСОК ЦИТИРОВАННОЙ ЛИТЕРАТУРЫ}

[1] Brun V. Le crible d'Eratosthene et le theoreme de Goldbach // C. R. Acad. Sci. Paris. 1919. V. 168. P. 544-546.

[2] Selberg A. On elementary methods in prime number theory and their limitations // II. Scand. Mat. Kongr. Trondheim. 1949. P. 13-22.

[3] Kuhn P. Zur Viggo Brunschen Siebmethode // I. Norske Vid. Selsk. Forh. Trondhjem. 1941. V. 14. № 39. P. 145-148.

[4] Бухштаб А. А. Комбинаторное усиление метода эратосфенова решета // УМН. 1967. T. 22. № 3 (135). C. 199-226.

[5] Richert H.-E. Selberg's sieve with weights // Mathematika. 1969. V. 16. № 31. P. 1-22.

[6] Laborde M. Buchstabs sifting weights // Mathematika. 1979. V. 26. P. 250-257.

[7] Бухштаб А. А. Новый тип весового решета // Тезисы докл. всес. конф. "Теория чисел и ее приложения". Тбилиси. 1985. С. 22-24.

[8] Halberstam H., Richert H.-E. Sieve methods. London: Acad. Press., 1974.

[9] Вахитова Е. В. О некоторых приложениях одномерного решета с весами // Матем. заметки. 1992. Т. 51. № 6. С. 139-141.

[10] Вахитова Е.В. О приложении функций Бухштаба // Матем. заметки. 1995. Т. 57. №1. C. $121-125$.

[11] Левин Б. В., Тимофеев Н. М. Распределение арифметических функций в среднем по прогрессиям (теоремы типа Виноградова-Бомбьери) // Матем. сб. 1984. Т. 125 (167). № 4(12). C. $558-572$. 